\title{
Precise Characterization and Multiobjective Optimization of Low Noise Amplifiers
}

\author{
Josef DOBES, Jan MICHAL, Jakub POPP, David CERNY, Martin GRABNER, \\ Frantisek VEJRAZKA, Jakub KAKONA, Stepan MATEJKA
}

Department of Radioelectronics, Czech Technical University in Prague, Technická 2, 16627 Praha, Czech Republic

dobes@fel.cvut.cz, jan.michal@yahoo.com, poppjaku, cernyda2@fel.cvut.cz, mgrabner@cmi.cz, vejrazka,kakonjak,matejka@fel.cvut.cz

\begin{abstract}
Although practically all function blocks of the satellite navigation receivers are realized using the CMOS digital integrated circuits, it is appropriate to create a separate low noise antenna preamplifier based on a low noise pHEMT. Such an RF front end can be strongly optimized to attain a suitable tradeoff between the noise figure and transducer power gain. Further, as all the four principal navigation systems (GPS, GLONASS, Galileo, and COMPASS) work in similar frequency bands (roughly from 1.1 to $1.7 \mathrm{GHz}$ ), it is reasonable to create the low noise preamplifier for all of them. In the paper, a sophisticated method of the amplifier design is suggested based on multiobjective optimization. A substantial improvement of a standard optimization method is also outlined to satisfy a uniform coverage of Pareto front. Moreover, for enhancing efficiency of many times repeated solutions of large linear systems during the optimization, a new modification of the Markowitz criterion is suggested compatible with fast modes of the LU factorization. Extraordinary attention was also given to the accuracy of modeling. First, an extraction of pHEMT model parameters was performed including its noise part, and several models were compared. The extraction was carried out by an original identification procedure based on a combination of metaheuristic and direct methods. Second, the equations of the passive elements (including transmission lines and T-splitters) were carefully defined using frequency dispersion of their parameters as $Q$, ESR, etc. Third, an optimal selection of the operating point and essential passive elements was performed using the improved optimization method. Finally, the s-parameters and noise figure of the amplifier were measured, and stability and third-order intermodulation products were also checked.
\end{abstract}

\section{Keywords}

Low noise amplifier, noise figure, transducer power gain, pHEMT, CAD, multiobjective optimization, goal attainment method, Pareto front, Markowitz criterion

\section{Introduction}

The optimization algorithms generally require strong convergence behavior and adequate computational efficiency.
In state-of-the-art optimization procedures, these properties are mostly searched as a combination of more reliable, but slower metaheuristic algorithms [1-6] with faster, but less stable direct optimization methods [7-10]. We have suggested an original combination of these methods in a robust three-step model identification procedure, which has been used for necessary extraction of pHEMT model parameters. Moreover, for the multiobjective optimization, a uniform coverage of the Pareto front [11] is also very important, especially for multidimensional tasks. We have used our improvement of the goal attainment method [9], which covers the Pareto front at least asymptotically uniformly and enables better automation of the procedure.

The LU factorization is considered the most demanding part of the circuit analysis from the computational point of view, especially in the case of many times repeated solutions during the multiobjective optimization. The elimination process during the LU factorization creates nonzero entries at positions, which correspond to zeros in the original matrix. The fill-in is the set of all the entries, which were originally zeros and took on nonzero values at any step of the factorization. The fill-in is able to highly increase the memory requirement, and the total computational time correspondingly. Generally usable tools $[12,13]$ do not address this problem because they operate with regular matrices only.

For solving this problem, the heuristic Markowitz criterion is still widely used [14-16]. The Markowitz criterion prevents expressions from swelling in a sparse matrix by selecting a pivot such that the number of entries which remain zero during the elimination is maximal, i.e., the fill-in created by the factorization is minimal. However, the Markowitz criterion is incompatible with a wide class of the fast algorithm modes for the LU factorization because a selection of the pivot from a whole submatrix is not allowed (the fast LU factorization only allows the pivot selection from a row or column). Therefore, the Markowitz criterion must be modified correspondingly for the fast LU factorization algorithm.

At present, there are many pHEMT models available in CAD tools. Their precision is often compared in references in a detailed way [17-20]. We have identified more types of the models used in low noise amplifiers, and we selected the one as a compromise between its accuracy and complexity. 


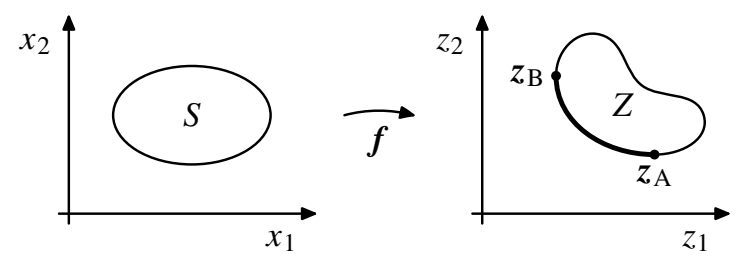

Fig. 1. Feasible region $(S)$, feasible objective region $(Z)$, and Pareto front.

\section{Multiobjective Optimization}

Any circuit optimization subject to multiple, mostly mutually contradictory requirements represents so-called multiobjective optimization problem. A comprehensive description of the procedure for solving the multiobjective optimization problem can be found in [21]; in this section, a brief but improved and a bit more precise definition will be given.

\subsection{The Multiobjective Optimization Problem}

The multiobjective optimization problem can be formally written in the standard form of a minimization:

$$
\underset{\boldsymbol{x} \in S}{\operatorname{minimize}}\left\{f_{1}(\boldsymbol{x}), f_{2}(\boldsymbol{x}), \ldots, f_{k}(\boldsymbol{x})\right\}
$$

Vectors of design variables $\boldsymbol{x}$ belong to a feasible region $S, S \subseteq \mathfrak{R}^{n}$, which can be specified by several equality constraints, inequality constraints, and/or by bounding allowed intervals for design variables $x_{i}$. Let us denote $\boldsymbol{f}(\boldsymbol{x})=\left(f_{1}(\boldsymbol{x}), f_{2}(\boldsymbol{x}), \ldots, f_{k}(\boldsymbol{x})\right)^{\top}$ the vector of objective functions and $Z=f(S)$ the image of the feasible region.

\subsection{Pareto Optimality}

Because the applied constraints are opposing one another, it is impossible to minimize them all simultaneously. Some tradeoffs need to be made, which leads to the concepts of Pareto optimality and noninferiority. A noninferior solution is one, whose any component can be further improved only at the cost of deteriorating another one. The set of noninferior solutions is called Pareto front, illustrated in Fig. 1 as the thicker part of the curve between points $z_{\mathrm{A}}$ and $z_{\mathrm{B}}$. By solving the problem (1) is then understood the obtaining of a sufficient number of noninferior solutions covering part of the Pareto front with a sufficient density (and uniformity).

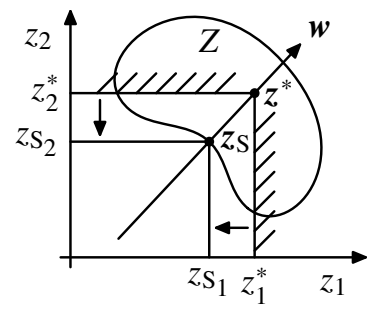

Fig. 2. Geometrical representation of the (unmodified original) Goal Attainment Method.

\section{3 (Standard) Goal Attainment Method}

The classical (unmodified) Goal Attainment Method (GAM) [7-9] solves the multiobjective optimization problem by solving the singleobjective problem

$$
\begin{array}{cl}
\underset{\gamma \in \mathfrak{R}, \boldsymbol{x} \in S}{\operatorname{minimize}} & \gamma \\
\text { subject to } & f_{i}(\boldsymbol{x})-w_{i} \gamma \leqq z_{i}^{*}, i=1, \ldots, k,
\end{array}
$$

in which $f_{i}$ are the individual design goals to be minimized, $S$ is the feasible solution set (feasible region), $z_{i}^{*}$ are some predetermined reference design goal values corresponding to $f_{i}, w_{i}, w_{i} \in \mathfrak{R}$ are predetermined weighting coefficients and $\gamma$ is an artificially introduced variable representing the single objective function.

GAM thus needs $2 k$ parameters $z_{i}^{*}$ and $w_{i}$ provided by the user, although only $2 k-1$ degrees of freedom determine the obtained result, as can be seen from the $2 \mathrm{D}$ example in Fig. 2. The objective vector $z^{*}=\left(z_{1}^{*}, z_{2}^{*}\right)^{\top}$ represents a pivot point in the objective space through which the $z_{\mathrm{S}}$ point moves in the direction of $\boldsymbol{w}=\left(w_{1}, w_{2}\right)^{\top}$ while varying $\gamma$. Therefore only the direction of the vector $\boldsymbol{w}$ is significant.

\subsection{Suggested Method for an Assymptotically Uniform Coverage of the Pareto Front}

Our proposed method further enhances the classical Goal Attainment Method with automatic generation of the predetermined parameters. A normalization is used instead of applying a weighting vector:

$$
\underset{\boldsymbol{x} \in S}{\operatorname{minimize}} \max _{i=1, \ldots, k} \frac{f_{i}(\boldsymbol{x})-z_{i}^{*}}{z_{i}^{\text {nad }}-\bar{z}_{i}}
$$

where $\bar{z}_{i}$ represent the components of the ideal vector $\bar{z}=\left(\bar{z}_{1}, \bar{z}_{2}, \ldots, \bar{z}_{k}\right)^{\top}$ found by independent minimization of each objective function

$$
\bar{z}=\left[\min _{\boldsymbol{x} \in S} f_{1}(\boldsymbol{x}), \min _{\boldsymbol{x} \in S} f_{2}(\boldsymbol{x}), \ldots, \min _{\boldsymbol{x} \in S} f_{k}(\boldsymbol{x})\right]^{\top},
$$

$z_{i}^{\text {nad }}$ compose the nadir objective vector $z^{\text {nad }}$ and they are the maximum (i.e., the worst-case) values of corresponding components $\left(\bar{z}_{i}\right)_{j}$ resulting from the independent minimization:

$$
z^{\text {nad }}=\left[\max _{i}\left(\bar{z}_{i}\right)_{1}, \ldots, \max _{i}\left(\bar{z}_{i}\right)_{k}\right]^{\top} .
$$

A uniform coverage of the Pareto front is secured by a pseudorandom generation of the pivot vector $z^{*}$ in such a way that it uniformly covers a preselected region in the $Z$ space. The random generation of the reference set can be performed as follows: starting with $k$ vertices $z_{0,1}, z_{0,2}, \ldots, z_{0, k}$ and a $(k-1)$-tuple of uniformly distributed and independent random numbers $r_{i} \in[0,1)$ for $i=1, \ldots, k-1$, this sequence of assignments will be performed to calculate a point $\boldsymbol{z}_{k-1,1}$ :

$$
\begin{aligned}
\text { for } i & :=1 \text { to } k-1 \text { do } \\
\text { begin } t_{i} & :=\sqrt[k-i]{r_{i}} \\
& \text { for } j:=1 \text { to } k-i \text { do } \\
\quad z_{i, j} & :=\left(1-t_{i}\right) z_{i-1,1}+t_{i} z_{i-1, j+1}
\end{aligned}
$$


This procedure calculates a total of $N=\sum_{i=1}^{k-1} i=k(k-1) / 2$ points $z_{i, j}$ in the $k$-dimensional objective space (i.e., $N=1$, 3 , and 6 points for $k=2,3$, and 4) from $k$ initially known vertices of the reference set. Much more details about this procedure can be found in [21], and a mathematical proof of the asymptotic uniformity of the coverage is given in [22].

\section{Modifying the Markowitz Criterion}

Precise modeling of RF devices generally leads to a number of transmission lines, vias, T-splitters, and parasitic inductors and capacitors to be included to a circuit model. Therefore, the matrix of the system created in the DC, AC, and transient analyses could be quite large, and the LU factorization of the (Jacobian) matrix should be arranged to suppress the fill-in (the set of all the entries, which were originally zeros and took on nonzero values at any step of the LU factorization). For solving this problem, the heuristic Markowitz criterion is still widely used [14-16]. The Markowitz criterion prevents expressions from swelling in a sparse matrix by selecting a pivot such that the number of entries which remain zero during the elimination is maximal, or phrased the other way around, that the fill-in created by the operation is minimal. However, the Markowitz criterion is incompatible with a wide class of the fast algorithm modes for the LU factorization [23] because a selection of the pivot from a whole submatrix is not allowed (the algorithm modifications for the fast LU factorization only allow the pivot selection from a row or a column). Therefore, the Markowitz criterion must be modified correspondingly for an efficient implementation of the (fast) LU factorization algorithm.

\subsection{Classical Form of the Markowitz Criterion}

The algorithm itself is not too complicated [15]. Suppose the LU factorization of $\boldsymbol{A}$ has proceeded through the first $k$ stages. For each $i^{\text {th }}$ row in the active $(m-k) \times(m-k)$ submatrix, let $r_{i}^{(k)}$ denote the number of entries. Similarly, let $c_{j}^{(k)}$ denote the number of entries in the $j^{\text {th }}$ column. The Markowitz criterion is to select as pivot the entry $A_{i j}^{(k)}$ from the $(m-k) \times(m-k)$ submatrix that satisfies

$$
\min _{i, j}\left(r_{i}^{(k)}-1\right)\left(c_{j}^{(k)}-1\right) \text {. }
$$

The usage of this entry as the searched pivot causes $\left(r_{i}^{(k)}-1\right)\left(c_{j}^{(k)}-1\right)$ entry modifications at the $k^{\text {th }}$ step. Not all these modifications will result in fill-in - therefore, the Markowitz criterion is actually only an approximation to the choice of the pivot which introduces the least fill-in.

\subsection{Simplest Fast Mode Compatible Criterion}

The entry modifications as a conclusion of applying the Markowitz weights (7) are incompatible with the fast LU factorization algorithm because the matrix structure changes in every stage. Therefore, remembering the structure of the nonzero elements could not have any practical sense under these conditions. For this reason, we should use a criterion that does not change the matrix structure during the LU factorization provided that the pivots remain relatively weighty in comparison with the other matrix elements. The simplest algorithm is natural. The total numbers of the potentially nonzero elements are determined for all the matrix columns. After that, the columns are reordered according to these numbers - the first and last should be those with the minimum and maximum numbers of such elements, respectively.

\subsection{Modified Form of the Markowitz Criterion}

A more sophisticated criterion can be created on the basis of idea of the Markowitz weights (7). However, they must be used in another way compatible with the fast LU factorization algorithm. To each potentially nonzero element located in $i^{\text {th }}$ row and $j^{\text {th }}$ column, the weight is assigned equal to the product of the numbers of other potentially nonzero elements in the $i^{\text {th }}$ row $r_{i}-1$ and $j^{\text {th }}$ column $c_{j}-1\left(r_{i}\right.$ and $c_{j}$ are the numbers of the nonzero elements in the $i^{\text {th }}$ row and $j^{\text {th }}$ column, respectively)

$$
\begin{aligned}
W_{i j}= & \begin{cases}\left(r_{i}-1\right)\left(c_{j}-1\right) & \text { for potentially nonzero element } \\
0 & \text { for element which is always zero, }\end{cases} \\
& i=1, \ldots, m, j=1, \ldots, m
\end{aligned}
$$

The weight $W_{i j}$ represents an estimation of the fill-in addition that can arise if the $(i, j)$ element becomes a pivot. The next part of the procedure differs from that in (7) because the column orientation of the LU factorization [23] must be held. Instead of an immediate reordering in the matrix, the average weights are determined for all columns using the element weights (8)

$$
w_{j}=\frac{1}{c_{j}} \sum_{\substack{i=1, \ldots, m \wedge A_{i j} \neq 0 \\(\forall \text { potentially nonzero elements) }}} W_{i j}, j=1, \ldots, m .
$$

Finally, the columns are reordered according to the weights (9) - the first and last should be those with the minimum and maximum weights, respectively.

\section{ATF-54143 Model Identification}

\subsection{Approximating the Scattering Parameters}

We have selected the Avago ATF-54143 low noise pHEMT [24], and we have identified its model using DC characteristics and $s$-parameters measured at a number of operating points [24]. We have extracted parameters of various nonlinear models (Statz, etc.) and carefully checked their accuracy. A typical comparison of the TriQuint TOM2 and Dobes (called Dobes in [17, 18]) models [17-20,25] is shown in Fig. 3. We have identified the models in the narrower frequency band from $1.1 \mathrm{GHz}$ to $1.7 \mathrm{GHz}$, because the suggested low noise preamplifier for the satellite navigation systems always works between these two frequencies. 

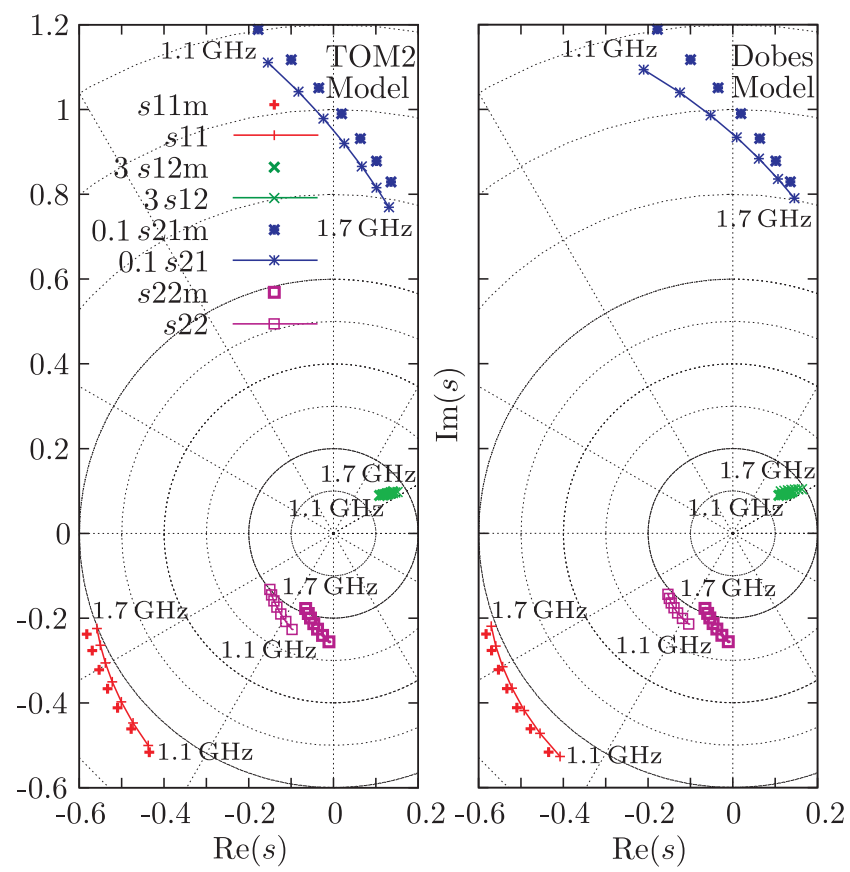

Fig. 3. Measured and from-model-generated $s$-parameters of the ATF-54143 pHEMT in the $s$-plane for the TOM2 and Dobes models for the frequencies from 1.1 to $1.7 \mathrm{GHz}$, $i_{\mathrm{d}}=60 \mathrm{~mA}$, and $v_{\mathrm{ds}}=3 \mathrm{~V}$. At this operating point, the Dobes model gives slightly more accurate $s_{11}$ and $s_{21}$.

For a quantified comparison of the models, we use the criterion

$$
\begin{gathered}
\mathrm{abs}_{s}=\frac{1}{4 m n} \sum_{i=1}^{2} \sum_{j=1}^{2} \sum_{k=1}^{m} \sum_{\ell=1}^{n}\left|\frac{s_{i j, k}^{\text {model }}\left(f_{\ell}\right)-s_{i j, k}^{\text {meas }}\left(f_{\ell}\right)}{s_{i j, k}^{\text {meas }}\left(f_{\ell}\right)}\right|, \\
\mathrm{rms}_{s}=\sqrt{\frac{1}{4 m n} \sum_{i=1}^{2} \sum_{j=1}^{2} \sum_{k=1}^{m} \sum_{\ell=1}^{n}\left|\frac{s_{i j, k}^{\text {model }}\left(f_{\ell}\right)-s_{i j, k}^{\text {meas }}\left(f_{\ell}\right)}{s_{i j, k}^{\text {meas }}\left(f_{\ell}\right)}\right|^{2}},
\end{gathered}
$$

where $m$ and $n$ are numbers of the operating points and frequencies at which the $s$-parameters were measured, respectively. In our case, $m=11$ and $n=7$, and the values of $\mathrm{abs}_{S}$ for the TOM2 and Dobes models were $3.58 \%$ and $3.73 \%$, respectively. In other words, the two models are comparable using (10a). However, the Dobes model was better using (10b): the values of $\mathrm{rms}_{s}$ for the TOM2 and Dobes models were $19.79 \%$ and $17.96 \%$, respectively. The reason consists in the $s_{22}$ accuracy, where the Dobes model was better in nine cases of eleven. (Let us emphasize that the accuracy of the $s_{22}$ parameter is well-known problem of the pHEMT models due to frequency dependence of output characteristics. Even contemporary models have this problem as can be seen in [26, Fig. 5], e.g.) Hence, the better $s_{22}$ precision was the reason why we selected the Dobes model. Other parameters were comparable, e.g., for $i_{\mathrm{d}}=60 \mathrm{~mA}$ and $v_{\mathrm{ds}}=3 \mathrm{~V}$ (it is shown in Fig. 3), the precisions of the $s_{11}$ and $s_{21}$ parameters were $3.09 \%$ and $7 \%$ for the TOM 2 model and (a bit better) $2.94 \%$ and $6.06 \%$ for the Dobes model, respectively.

\subsection{Modeling the Drain and Gate Noise Sources}

For the purpose of the maximal accuracy of the noise modeling, we have used the contemporary Curtice 3 model $[25,27]$. (The Curtice 3 model incorporates more other ones). The drain noise source is defined by semiclassical equation

$$
\left\langle i_{\mathrm{d}}^{2}\right\rangle=\left(4 k T g_{\mathrm{m}} P+4 k T g_{\mathrm{m}} P \frac{F_{n c}}{f}+K_{f} \frac{I_{\mathrm{ds}}^{A_{f}}}{f^{F_{f e}}}\right) \Delta f,
$$

where $k, g_{\mathrm{m}}, P, F_{n c}, K_{f}, A_{f}$, and $F_{f e}$ are Boltzman constant, transconductance, drain noise coefficient, flicker noise corner frequency, flicker noise coefficient, flicker noise exponent, and flicker noise frequency exponent, respectively.

The relatively novel part of the noise model is represented by gate noise source

$$
\left\langle i_{\mathrm{g}}^{2}\right\rangle=4 k T C_{\mathrm{gs}}^{2} \omega^{2} \frac{R}{g_{m}} \Delta f
$$

where $C_{\mathrm{gs}}$ and $R$ are zero bias gate-source junction capacitance and gate noise coefficient, respectively. There is also a correlation between the two noise sources (with a modified absolute temperature $T_{j}$ )

$$
\left\langle i_{\mathrm{g}}, i_{\mathrm{d}}^{*}\right\rangle=4 k T_{j} C_{\mathrm{gs}} \omega \sqrt{P R} C \Delta f
$$

where $C$ is gate-drain noise correlation coefficient; and the thermal noises generated by gate, source, and drain ohmic resistances $R_{\mathrm{g}}, R_{\mathrm{s}}$, and $R_{\mathrm{d}}$ are included, too:

$$
\left\langle i_{\mathrm{G}|\mathrm{S}| \mathrm{D}}^{2}\right\rangle=\frac{4 k T}{R_{\mathrm{g}|\mathrm{s}| \mathrm{d}}} \Delta f .
$$

The C.I.A. program [23] always contained (11) and (14) - however, for the accuracy purposes, the gate noise source model (12) has also been implemented. We have identified this model using datasheet [24], where some of the parameters are defined: $F_{n c}=1 \mathrm{MHz}, R=0.08 \Omega, P=0.2$, and $C=0.1$, and the exact values have been found by optimization comparing with the set of measured noise parameters.

\section{Using Multiobjective Optimization}

\subsection{Description of the Low Noise Preamplifier}

Let us mention a design of the low noise antenna preamplifier within the frequency range 1.1 to $1.7 \mathrm{GHz}$ in Fig. 4 (a standard circuit diagram), Fig. 5 (a simplified simulation schematic), and Figs. 6, 7, and 8 (a detailed circuit diagram used for the optimization). The terminal impedances were $50 \Omega$. The multiobjective optimization was used to simultaneously maximize the minimum transducer power gain $A_{\mathrm{pt}}$ over the whole frequency range, minimize the maximum noise figure $F_{n}^{\mathrm{dB}}$, while maintaining certain stability criteria and the quiescent operating point within chosen limits. The result is a set of noninferior solutions. The noninferior solution is the one in which an improvement in one objective in this case, $A_{\mathrm{pt}}$ or $F_{n}^{\mathrm{dB}}$ - requires a deterioration in another. 


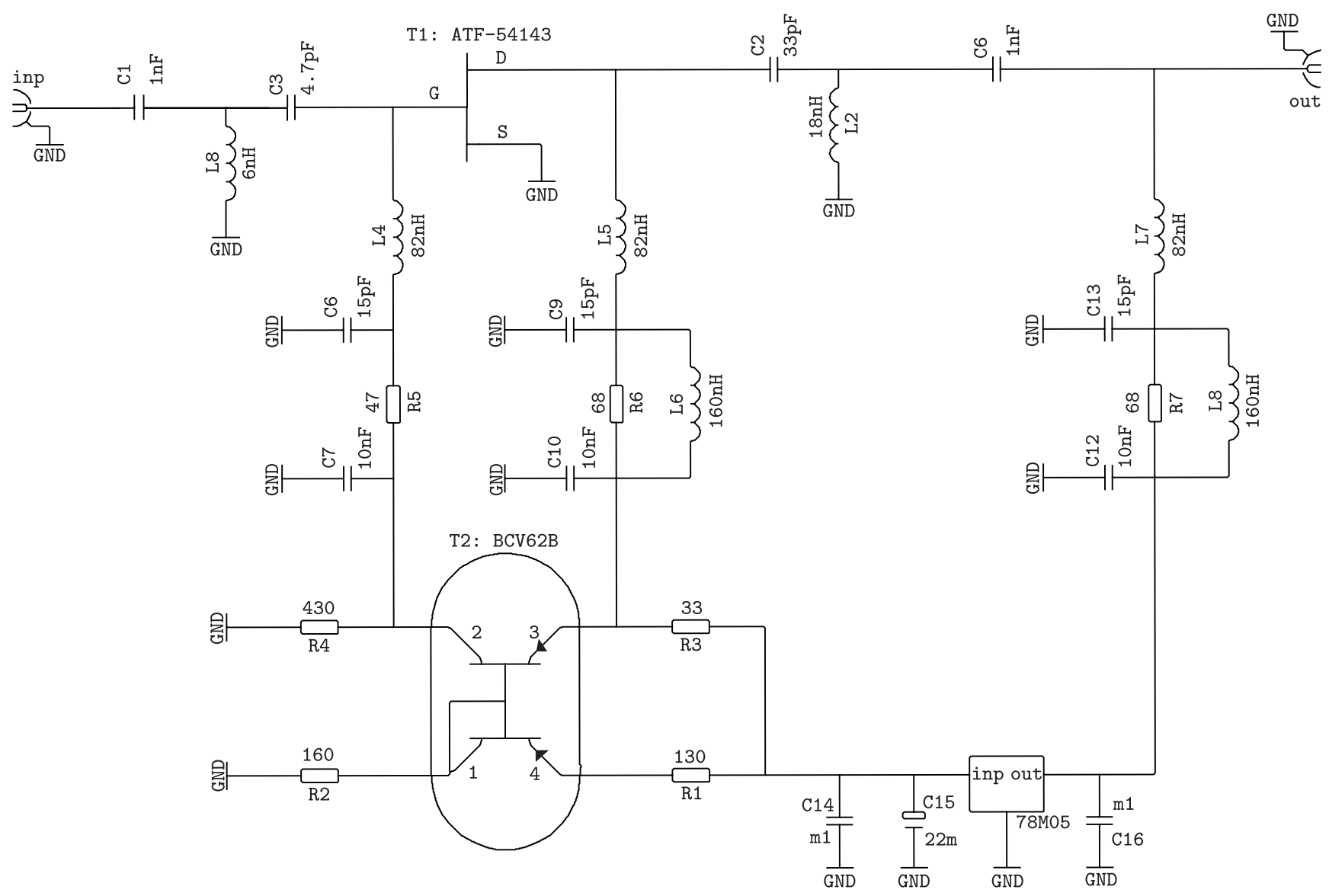

Fig. 4. Standard circuit diagram of the low noise antenna preamplifier for the multiconstellation GPS/Galileo/GLONASS/Compass receiver.

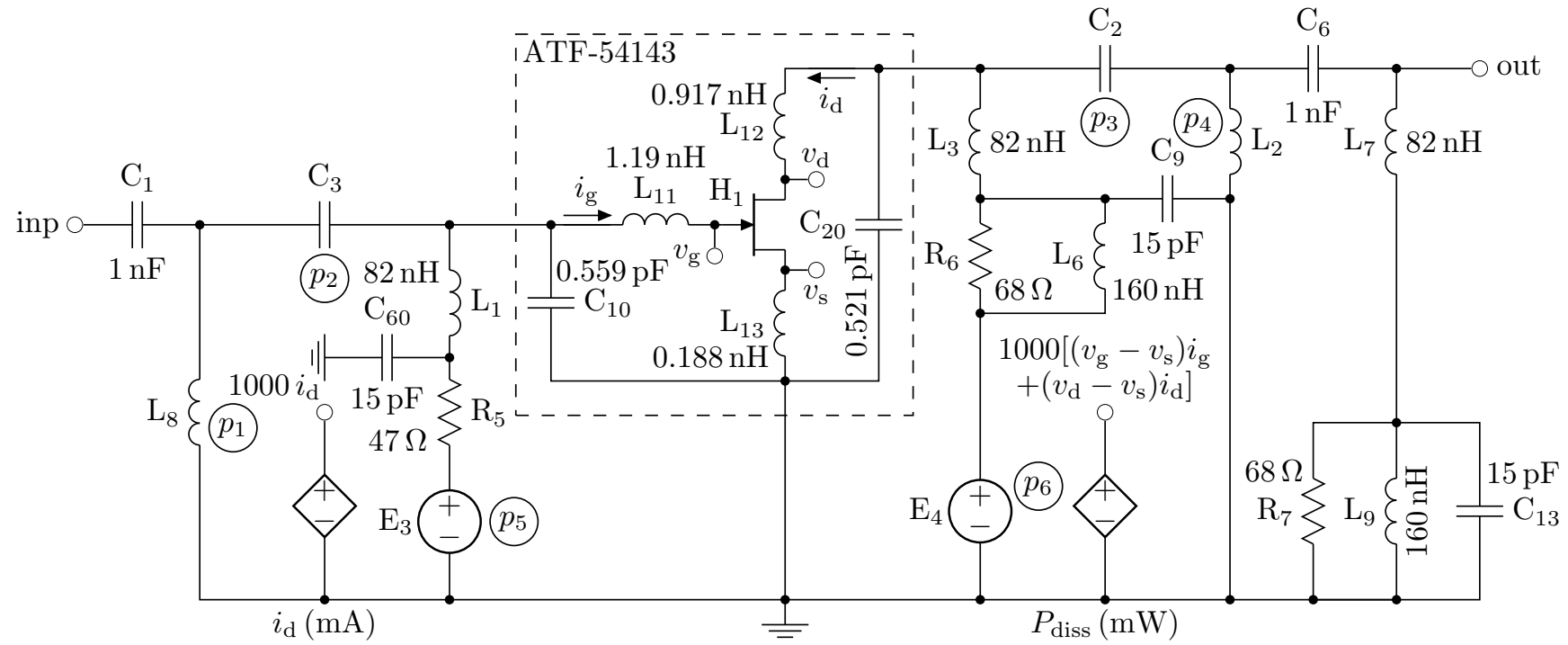

Fig. 5. Simplified simulation schematic of the low noise antenna preamplifier for the multiconstellation GPS/Galileo/GLONASS/Compass receiver. Actual power supply is brought through the output port and diverted by choke L7. The actual biasing circuitry that transfers it to the places of voltage sources E3 and E4 is not part of the simulation. Nuances like intermediate transmission line segments, T splitters or component models have been omitted here. The controlled sources are used for checking the optimization constraints $i_{\mathrm{d}}$ and $P_{\text {diss }}$. The circles mark the six circuit parameters $p_{1}, \ldots, p_{6}\left(L_{8}, C_{3}, C_{2}, L_{2}, E_{3}\right.$, and $\left.E_{4}\right)$ to be determined by the multiobjective optimization. 


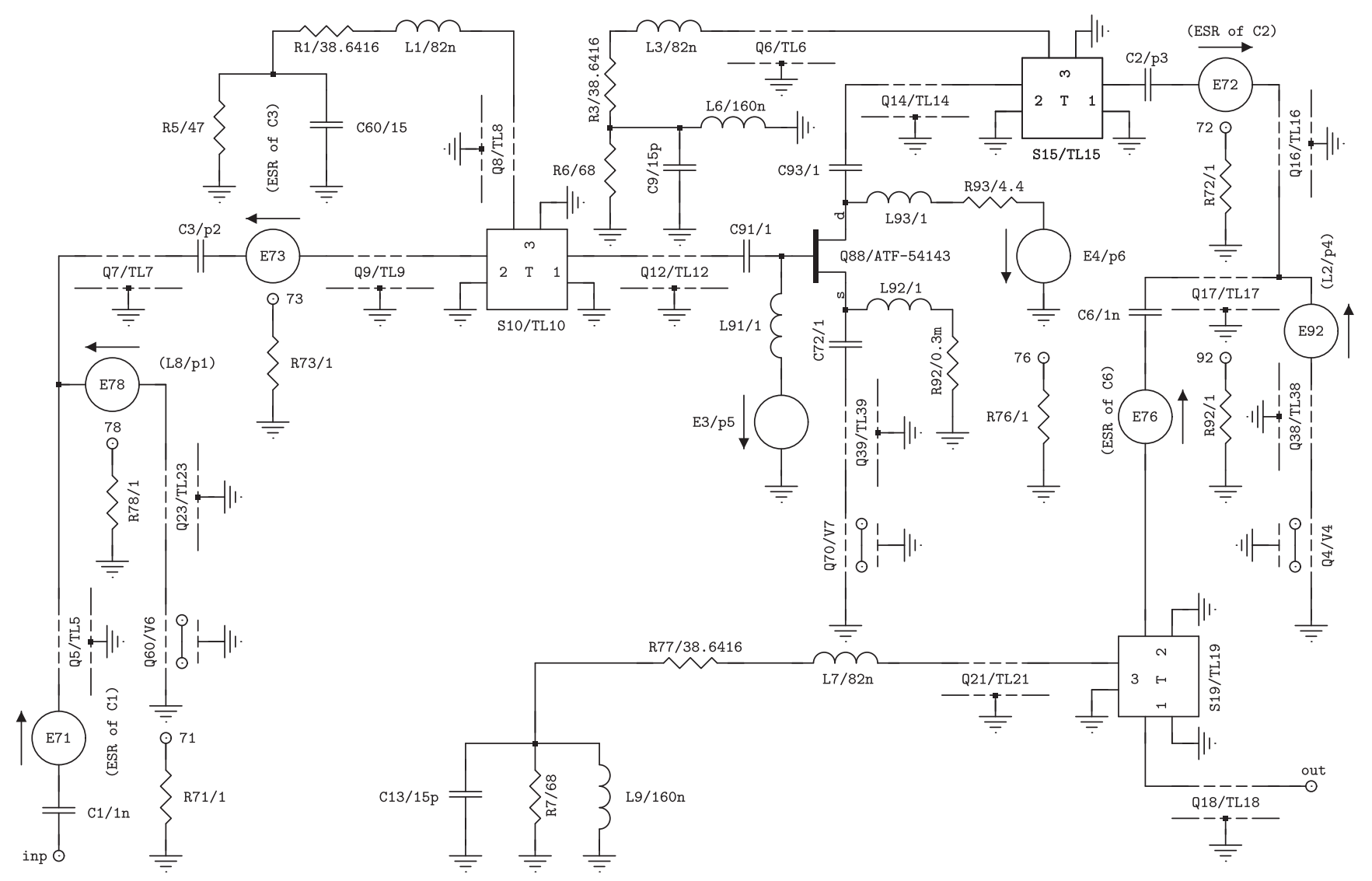

Fig. 6. Detailed simulation schematic. Transmission line segments and T-splitters are specified with sets of frequency dependent $s$-parameters, the former also being supplemented with frequency dependent noise sources. Biasing and power supply branches with chokes L1, L3 and L7 are not used for setting the DC operating point as the $s$-parameter specified blocks do not work appropriately for the DC analysis in the C.I.A. simulator, and also because the actual DC resistances involved are much different from the frequency dependent AC ones. Instead, ideal extremal capacitors C91-C93 and inductors L91-L93 are used to provide transistor biasing in DC analysis with correct resistance values without affecting AC behavior. Matching shunt coils L8 and L2 are modeled by means of controlled voltage sources E78 and E92 (as a function of the current through the inductor and its time derivative) providing inductance, series resistance and its noise contribution, all being nominal value and frequency dependent and based on linearly interpolated catalog data for the equivalent inductance and quality factor. Chokes L1, L3 and L7, which have only a minor effect on AC behavior, are left constant, with constant series resistances R1, R3 and R77 based on an assumed quality factor of 20 at $1.5 \mathrm{GHz}$. Series capacitances in the signal path C1, C3, C2 and C6 are supplemented with a frequency dependent ESR provided by controlled voltage sources E71, E73, E72 and E76. One-ohm resistances present in the schematic serve as sources of reference noise voltage used by the controlled sources. Transmission line segments marked as ' $\mathrm{V}$ ' represent PCB vias providing ground connections. Detailed noise models of the pHEMT, transmismission lines and vias are shown in Figs. 7 and 8.

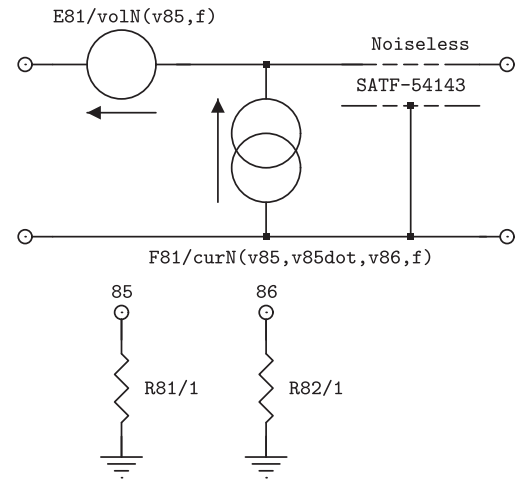

Fig. 7. The transistor noise model is composed of partially correlated sources of noise voltage and noise current placed at the input of the original (noise-free) $s$-parameter specified twoport. The noise current is a combination of three components: two fully correlated with the noise voltage (inphase and quadrature) and an uncorrelated third one based on the noise of another reference resistor.

\subsection{Description of the Requested Design Goals}

The topology is a natural single stage application of pHEMT in the common source configuration with LC impedance matching networks at the input and the output. There are six design parameters: four component values of $L_{8}, C_{3}, C_{2}$ and $L_{2}$, and two bias voltages, gate $E_{3}$ and drain $E_{4}$ as shown in Fig. 5. Design goals are also six; they are shown in Tab. 1: the above mentioned two objectives, $A_{\mathrm{pt}}$ and $F_{n}^{\mathrm{dB}}$, and four inequality constrained goals, the maximum drain current $i_{\mathrm{d}}$, drain-source voltage $v_{\mathrm{ds}}$, Rollett stability factor $K_{\mathrm{Rs}}$, and the determinant of the $s$-matrix $|\Delta|$. The absolute stability criteria $K_{\mathrm{Rs}}$ and $|\Delta|$ were still used, even if the transistor's parameters did not allow for achieving a full unconditional stability $\left(K_{\mathrm{Rs}}>1,|\Delta|<1\right)$, and had to be relaxed to the region of potential instability $\left(K_{\mathrm{Rs}}>0.5,|\Delta|<0.9\right)$. 


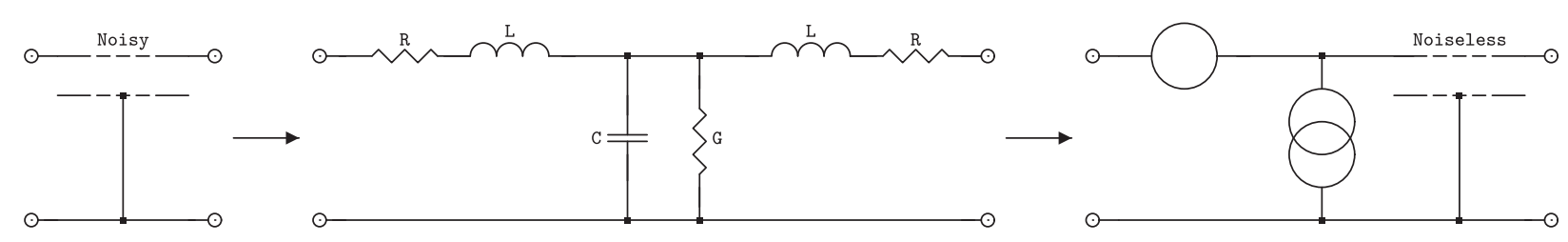

Fig. 8. The used noise model of short transmission line segment injects the collective noise of the R and $\mathrm{G}$ parts of an equivalent symmetrical T-structure, transferred to the input as noise voltage and noise current.

\begin{tabular}{|c|c|c|c|c|c|}
\hline No. & Symbol & Type & Direction & $\begin{array}{c}\text { Optimum/ } \\
\text { Bound }\end{array}$ & Unit \\
\hline 1 & $A_{\mathrm{pt}}$ & Objective & Maximum & $\geqq 15.5$ & $\mathrm{~dB}$ \\
2 & $F_{n}^{\mathrm{dB}}$ & Objective & Minimum & $\leqq 2$ & $\mathrm{~dB}$ \\
3 & $i_{\mathrm{d}}$ & Constraint & $\leqq$ & 75 & $\mathrm{~mA}$ \\
4 & $v_{\mathrm{ds}}$ & Constraint & $\leqq$ & 3.75 & $\mathrm{~V}$ \\
5 & $K_{\mathrm{Rs}}$ & Constraint & $\geqq$ & 0.5 & - \\
6 & $|\Delta|$ & Constraint & $\leqq$ & 0.9 & - \\
\hline
\end{tabular}

Tab. 1. Design goals for the antenna low noise preamplifier.

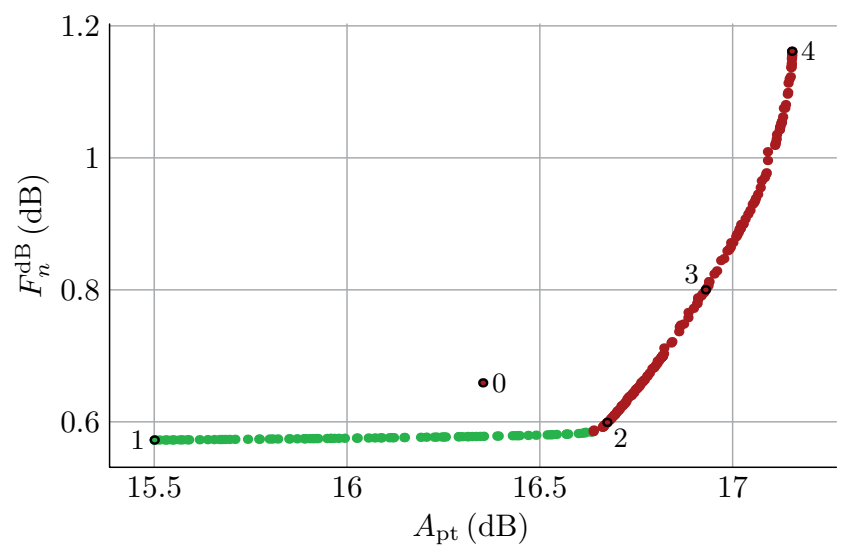

Fig. 9. Computed Pareto front in the objective space $A_{\mathrm{pt}}, F_{n}^{\mathrm{dB}}$. The dot marked with 0 represents the original unoptimized reference design. Solutions marked with $1-4$ were selected as the representatives with the results shown in Tab. 2 and Fig. 11. They were numbered from the lowest $A_{\mathrm{pt}}$ of $15.5 \mathrm{~dB}$ to the highest $A_{\mathrm{pt}}$ of $17.2 \mathrm{~dB}$. The green dots (those for $A_{\mathrm{pt}} \leqq 16.635$ ) mark the part of the Pareto front with $K_{\mathrm{Rs}}>1$, the red dots (including the original 0 ) mark the part of the Pareto front with $K_{\mathrm{Rs}} \leqq 1$. (Let us also note the uniformity of the Pareto front covering.)

\subsection{Description of the Obtained Design Results}

Totally 262 solutions have been obtained, spanning the whole part of the Pareto front selected by the requirements $A_{\mathrm{pt}} \geqq 15.5 \mathrm{~dB} \wedge F_{n}^{\mathrm{dB}} \leqq 2 \mathrm{~dB}$ as shown in Fig. 9. There were no solutions found with $A_{\mathrm{pt}}$ above $17.2 \mathrm{~dB}$. Tab. 2 shows a selection of four representative solutions. All have their $E_{4}$ (i.e., $v_{\mathrm{DD}}$ ) at the chosen upper bound value of $3.75 \mathrm{~V}$. Finally, Fig. 11 shows plots for the four selected solutions as well as for the original unoptimized circuit (marked with 0 ). It is obvious the multi-objective optimization improved the circuit. For example, the tested solution No. 2 gives better noise figure for all frequencies, and a comparable and more uniform frequency dependence of the transducer power gain.

\begin{tabular}{|c|c|c|c|c|c|c|c|}
\hline \multirow[b]{2}{*}{ No. } & \multirow[b]{2}{*}{ Symbol } & \multirow{2}{*}{$\begin{array}{l}\text { Original } \\
\text { Value: } 0\end{array}$} & \multicolumn{4}{|c|}{ Solution Number } & \multirow[b]{2}{*}{ Unit } \\
\hline & & & 1 & 2 & 3 & 4 & \\
\hline 1 & $L_{8}$ & 6.8 & 5.78 & 6.18 & 4.93 & 4.15 & $\mathrm{nH}$ \\
\hline 2 & $C_{3}$ & 4.7 & 6.2 & 5.62 & 4.46 & 3.63 & $\mathrm{pF}$ \\
\hline 3 & $C_{2}$ & 33 & 1.64 & 5.74 & 7.97 & 200 & $\mathrm{pF}$ \\
\hline 4 & $L_{2}$ & 18 & 6.94 & 7.2 & 7.01 & 11.2 & $\mathrm{nH}$ \\
\hline 5 & $E_{3}$ & 0.602 & 0.595 & 0.57 & 0.567 & 0.552 & V \\
\hline 6 & $E_{4}$ & 3.26 & 3.75 & 3.75 & 3.75 & 3.75 & V \\
\hline 1 & $A_{\mathrm{pt}}$ & 16.4 & 15.5 & 16.7 & 16.9 & 17.2 & $\mathrm{~dB}$ \\
\hline 2 & $F_{n}^{\mathrm{dB}}$ & 0.659 & 0.572 & 0.599 & 0.8 & 1.16 & $\mathrm{~dB}$ \\
\hline 3 & $i_{\mathrm{d}}$ & 60 & 62.9 & 54.3 & 53.2 & 48.3 & $\mathrm{~mA}$ \\
\hline 4 & $v_{\mathrm{ds}}$ & 3 & 3.47 & 3.51 & 3.52 & 3.54 & $\mathrm{~V}$ \\
\hline 5 & $K_{\mathrm{Rs}}$ & 0.986 & 1.04 & 0.988 & 0.991 & 0.98 & - \\
\hline 6 & $|\Delta|$ & 0.686 & 0.539 & 0.685 & 0.734 & 0.737 & - \\
\hline 7 & $P_{\text {diss }}$ & 180 & 218 & 191 & 187 & 171 & $\mathrm{~mW}$ \\
\hline
\end{tabular}

Tab. 2. Four selected solutions from the Pareto front with their frequency characteristics shown in Fig. 11.

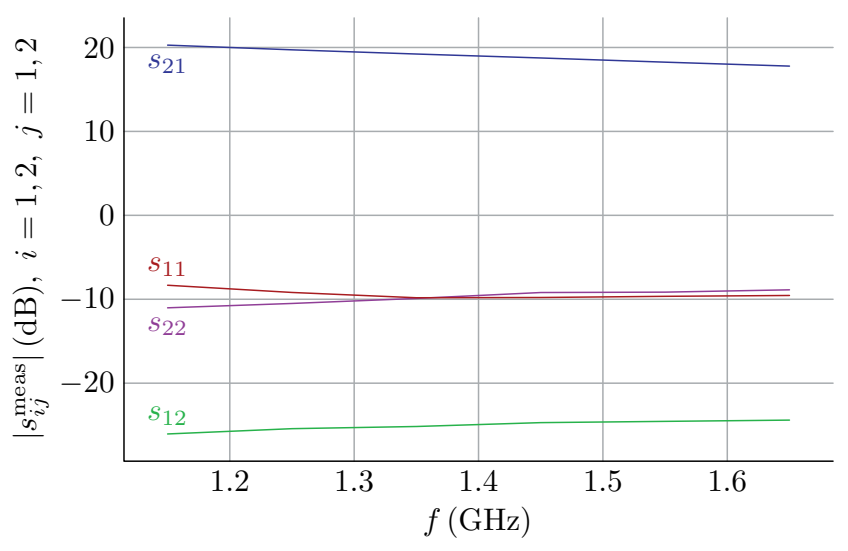

Fig. 10. Measured $s$-parameters of the low noise preamplifier.

\subsubsection{Measurement of the $s$-Parameters and Noise Figure}

The low noise preamplifier has been carefully measured and the results (as well as the typical data from Avago [24]) confirm a local minimum of the noise figure somewhere in the middle of the band: the unoptimized configuration (marked with 0 ) has the measured minimum at $1.225 \mathrm{GHz}$, the solution No. 2 (2) at $1.25 \mathrm{GHz}$. Moreover, the tested solution No. 2 gives better noise figure in the frequency band from approximately 1.1 to $1.7 \mathrm{GHz}$ as shown in Fig. 11. Moreover, the measured $s$-parameters of the circuit confirm the good amplifier's properties: both $s_{11}$ and $s_{22}$ are about $-10 \mathrm{~dB}$ in the frequency band 1.1-1.7 GHz as shown in Fig. 10 . 

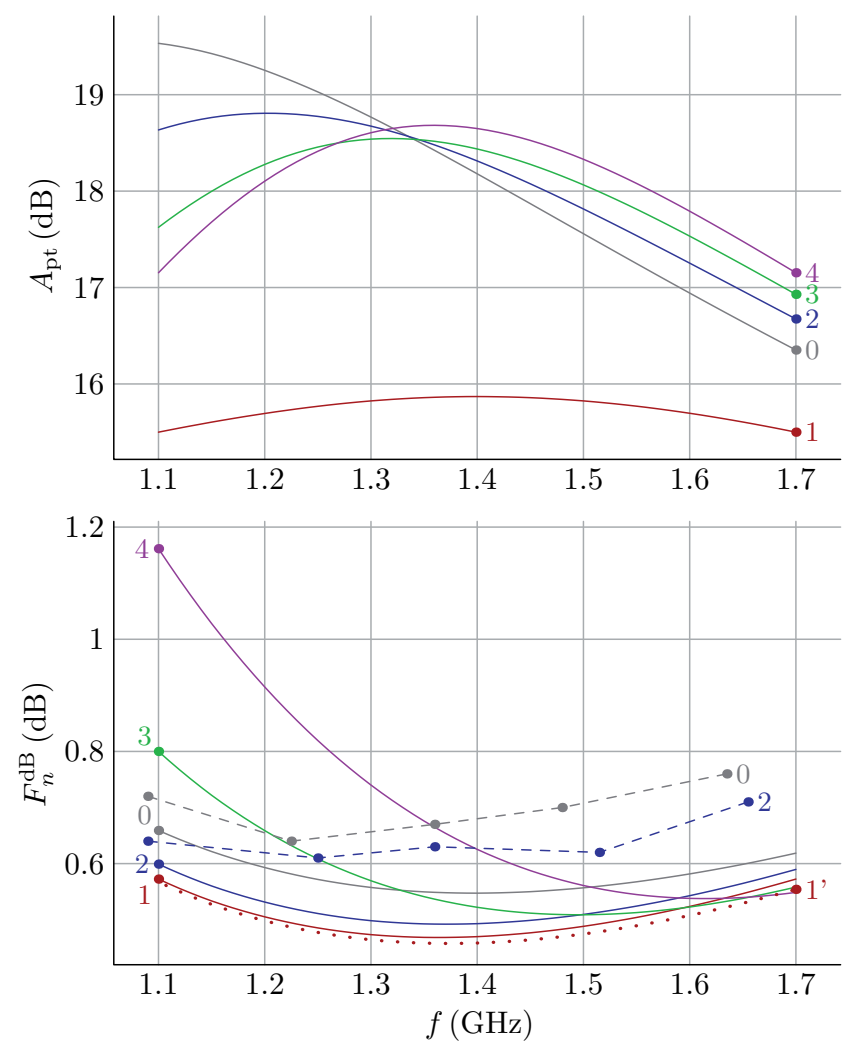

Fig. 11. Simulated (solid lines and the dotted one) and measured (dashed lines) $A_{\mathrm{pt}}$ and $F_{n}^{\mathrm{dB}}$ plots for the original (marked with 0 ) and four selected (marked with 1-4) solutions shown in Tab. 2. (The plot marked with 1' shows the noise figure plot in the case when the gate noise (12) is not included.) Let us emphasize that the (declared) error of the equipment for measuring the noise figure was (at least) $0.1 \mathrm{~dB}$; therefore, the difference between the original solution and the solution No. 2 is more important here than the obtained values themselves. (And this difference clearly shows that the algorithm has improved the noise figure comparing with the original solution. Further, we have detected an interference with an outer signal at some frequencies, and these measurements were excluded.)

\begin{tabular}{|c|l|l|c|}
\hline$f(\mathrm{GHz})$ & $|\Delta|$ & \multicolumn{1}{|c|}{$K_{\mathrm{Rs}}$} & $\mu$ \\
\hline 1.15 & 0.411 & 0.916 & 0.900 \\
1.25 & 0.417 & 0.929 & 0.916 \\
1.35 & 0.408 & 0.95 & 0.943 \\
1.45 & 0.406 & 0.932 & 0.926 \\
1.55 & 0.385 & 0.949 & 0.946 \\
1.65 & 0.368 & 0.958 & 0.958 \\
\hline
\end{tabular}

Tab. 3. Measured stability factors of the preamplifier.

\subsubsection{Checking the Low Noise Preamplifier Stability}

The low noise preamplifier stability has been checked by additional calculations and measurements. It is clear that the solution No. 1 is probably the most stable because its Rollett stability factor $K_{\mathrm{Rs}}$ shown in Tab. 2 is greater than one. For the tested solution No. 2 , the factors $|\Delta|, K_{\mathrm{Rs}}$, and $\mu$ have also been computed from measured data in a wide band analogically to [28, Fig. 5], and the values inside the satellite navigation band 1.1-1.7 GHz are shown in Tab. 3 .

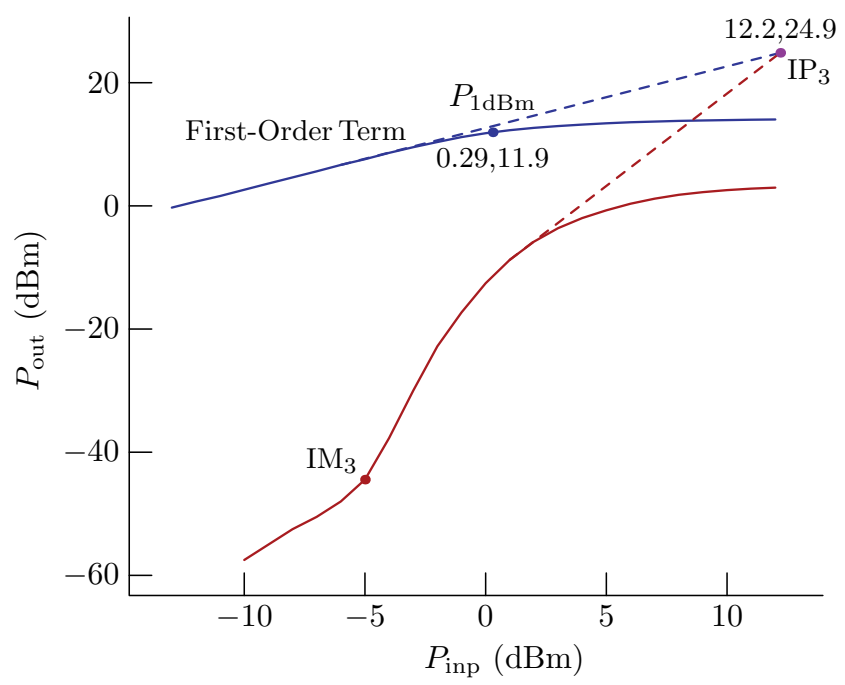

Fig. 12. Graphical post-processing of the $\mathrm{IP}_{3}$ measurement results for the original (unoptimized) amplifier (No. 0). Individual input tone frequencies $f_{1}$ and $f_{2}$ were 1.575 and $1.576 \mathrm{GHz}$, respectively. The $\left(f_{1} / f_{2}\right)$ level ratio was 1 . (This method of creating the $3: 1$ slope line led to a "worst-case" $\mathrm{IP}_{3}$ estimation.) Let us note the typical shape of the $\mathrm{IM}_{3}$ curve for the amplifiers with pHEMTs.

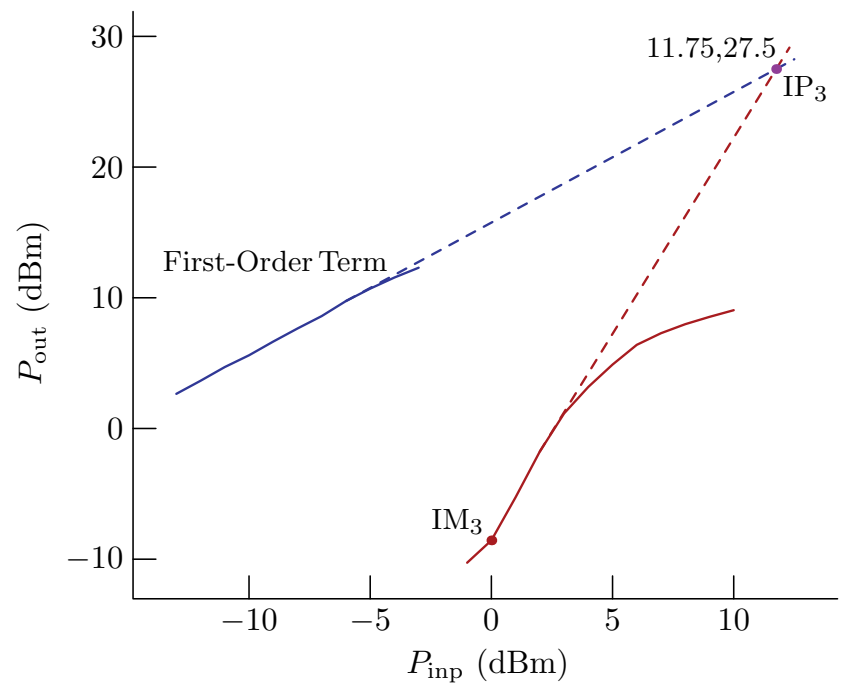

Fig. 13. Graphical post-processing of the $\mathrm{IP}_{3}$ measurement results for the solution No. 2. Individual input tone frequencies $f_{1}$ and $f_{2}$ were again 1.575 and $1.576 \mathrm{GHz}$, respectively. The $\left(f_{1} / f_{2}\right)$ level ratio was 1 , too.

\subsubsection{Checking the 3rd-Order Intermodulation Products}

We have also measured the third-order intermodulation products for the original unoptimized low noise preamplifier (marked with 0 in Figs. 9 and 11) and for the tested solution No. 2 (see the same figures), and the results are compared in Figs. 12 and 13. The measurements confirm improved properties of the solution No. 2 - let us emphasize better output/input dynamics, and, first of all, the better location of the $\mathrm{IP}_{3}$ point for the solution No. 2, comparable with [28]. 

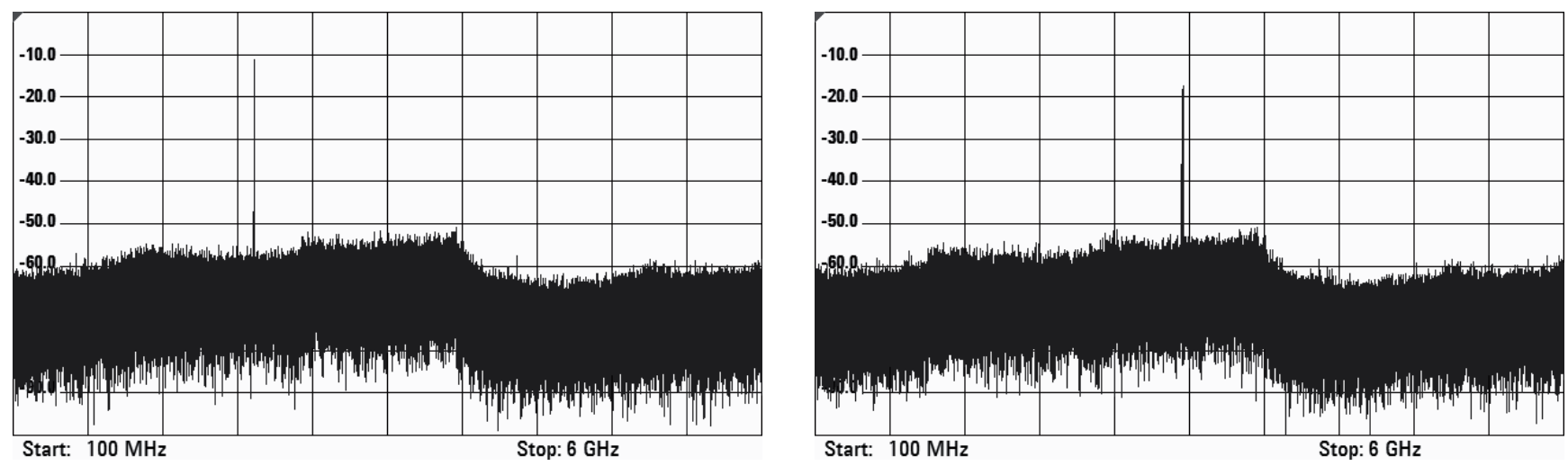

Fig. 14. Measured spectra of the low noise amplifier. The signal generator has been set to the constant level $-20 \mathrm{dBm}$. The spectra are shown for the case of the signal frequencies 2 and $3 \mathrm{GHz}$ (i.e., it could be clearly seen here how the output voltage decreases with the growing signal frequency - the spectrum was also measured at $1.5 \mathrm{GHz}$, and the exact result for the frequency $1.507777778 \mathrm{GHz}$ was $-10.7 \mathrm{dBm}$ ).

\begin{tabular}{|c|c|c|c|c|c|c|}
\hline \multirow{3}{*}{ Before LU Factorization } & \multicolumn{5}{|c|}{ After LU Factorization (average values) } \\
\cline { 2 - 7 } & \multicolumn{4}{|c|}{ DC Analysis } & \multicolumn{3}{c|}{ Frequency and Noise Analyses } \\
\cline { 2 - 7 } & Disarranged & Numb. in cols & Modif. Mark. & Disarranged & Numb. in cols & Modif. Mark. \\
\hline 1570 & $4154264.6 \%$ & $2826180.0 \%$ & $2604165.9 \%$ & $4340276.4 \%$ & $2739174.4 \%$ & $2566163.4 \%$ \\
\hline
\end{tabular}

Tab. 4. Comparison of the total number of nonzero matrix elements before and after the LU factorization for its tested three versions.

\subsubsection{Checking the Efficiency of the Modified Markowitz Criterion}

As the Jacobian matrix generated in the DC and frequency \& noise analyses of the circuit is quite large, we have also tested the criteria for suppressing the fill-in defined in Sec. 3, and the results are shown in Tab. 4. The worst results are clearly for classical (unmodified) LU factorization: although the matrix has 1570 nonzero elements before the LU factorization, this number increases to the values 4154 and 4340 after the LU factorization for the DC and frequency $\&$ noise analyses, respectively. If the simplest criterion for reordering the LU factorization described in Subsec. 3.2 is used, the results are better: the total numbers of the nonzero elements are 2826 and 2793 after the (correspondingly modified) LU factorization for the DC and frequency \& noise analyses, respectively. However, the best results are obtained using the proposed modified form of the Markowitz criterion defined in Subsec. 3.3: the monitored numbers are finally 2604 and 2566 for the DC and frequency \& noise analyses.

\section{Conclusions}

An improved algorithm for determining the tradeoff between the transducer power gain and noise figure of a low noise preamplifier was suggested. The modified algorithm is based on an improved Goal Attainment Method of the multiobjective optimization, which generates an asymptotically uniform coverage of the Pareto front. The efficiency of the whole procedure of the multiobjective optimization is increased using the implementation of the modified form of the Markowitz criterion. The complete design process is demonstrated using very accurate modeling the circuit active and passive elements followed by a reliable constrained optimization of the whole circuit. The final properties of the circuit including its stability were checked by various types of measurements in linear and nonlinear domains.

\section{Acknowledgments}

This work has been supported by the Technology Agency of the Czech Republic, grant No. TE01020186, and by the Grant Agency of the Czech Technical University in Prague, grant No. SGS15/161/OHK3/2T/13.

\section{References}

[1] ShORBAGy, M., MOUSA, A. A. A., FATHI, W. Hybrid Particle Swarm Algorithm for Multiobjective Optimization: Integrating Particle Swarm Optimization with Genetic Algorithms for Multiobjective Optimization. Saarbrücken (Germany): LAP (Lambert Academic Publishing), 2011. ISBN: 3847311492

[2] MOUSA, A. A. A. Study on Multiobjective Optimization Using Improved Genetic Algorithm: Methodology and Application. Saarbrücken (Germany): LAP (Lambert Academic Publishing), 2011. ISBN: 3846548898

[3] Tlelo-CUAUtle, E., GUERRA-GÓMEZ, I., DE la FRAGA, L. G., FLORES-BECERRA, G., POLANCO-MARTAGÓN, S., FAKHFAKH, M., REYES-GARCÍA, C. A., RODRÍGUES-GÓMEZ, G., REYES-SALGADO, G. Evolutionary algorithms in the optimal sizing of analog circuits. Intelligent Computational Optimization in Engineering, Studies in Computational Intelligence. Berlin (Germany): Springer, 2011, vol. 366, p. 109-138. ISBN: 9783642217043. DOI: 10.1007/978-3-642-21705-0_5 
[4] FAKHFAKH, M., SALLEM, A., BOUGHARIOU, M., BENNOUR, S., BRADAI, E., GADDOUR, E., LOULO,U M. Analogue circuit optimization through a hybrid approach. Intelligent Computational Optimization in Engineering, Studies in Computational Intelligence. Berlin (Germany): Springer, 2011, vol. 366, p. 297-327. ISBN: 9783642217043. DOI: 10.1007/978-3-642-21705-0_11

[5] HIRANO, H., YOSHIKAWA, T. A study on two-step search using global-best in PSO for multi-objective optimization problems. In Proceedings of the 6th International Conference on Soft Computing and Intelligent Systems/13th International Symposium on Advanced Intelligent Systems (SCIS/ISIS). Kobe (Japan), 2012, p. 1894-1897. DOI: 10.1109/SCIS-ISIS.2012.6505349

[6] CHIBA, K. Performance comparison of evolutionary algorithms applied to hybrid rocket problem. In Proceedings of the 6th International Conference on Soft Computing and Intelligent Systems/13th International Symposium on Advanced Intelligent Systems (SCIS/ISIS). Kobe (Japan), 2012, p. 1673-1678. DOI: 10.1109/SCISISIS.2012.6505130

[7] KOLO, B. Single \& Multiple Objective Optimization. Weatherford (Oklahoma, USA): Weatherford Press, 2011. ISBN: 161580014X

[8] COLLETTE, Y., SIARRY, P. Multiobjective Optimization: Principles and Case Studies. 2nd ed., rev. Berlin (Germany): Springer, 2004. ISBN: 9783642072833

[9] MIETTINEN, K. M. Nonlinear Multiobjective Optimization. 4th ed. New York (New York, USA): Springer Science \& Business Media, 2004. ISBN: 9781461375449

[10] MOOSAVIAN, S. A. A., GHAFARI, A., SALIMI, A., ABDI, N. Nonlinear multiobjective optimization for control of hydropower plants network. In Proceedings of the IEEE/ASME International Conference on Advanced Intelligent Mechatronics (AIM). Xi'an (China), 2008, p. 1278-1283. DOI: $10.1109 /$ AIM.2008.4601846

[11] MIYAZAKI, R., HAMADA, N., NAGATA, Y., ONO, I. A new Pareto frontier covering strategy in FS-MOGA for multi-objective function optimization. In Proceedings of the 6th International Conference on Soft Computing and Intelligent Systems/13th International Symposium on Advanced Intelligent Systems (SCIS/ISIS). Kobe (Japan), 2012, p. 1888-1893. DOI: 10.1109/SCIS-ISIS.2012.6505313

[12] ENGELN-MULLGES, G., UHLIG, F. Numerical Algorithms with C. Berlin (Germany): Springer, 1996. ISBN: 3540605304. DOI: 10.1007/978-3-642-61074-5

[13] PRESS, W. H., TEUKOLSKY, S. A., VETTERLING, W. T., FLANNERY, B. P. Numerical Recipes: The Art of Scientific Computing, 3rd ed., rev. New York (New York, USA): Cambridge University Press, 2007. ISBN: 9780521884075

[14] BALLARIN, C., KAUERS, M. Solving parametric linear systems: an experiment with constraint algebraic programming. ACM SIGSAM Bulletin, 2004, vol. 38, no. 2, p. 33-46. ISSN: 0163-5824. DOI: $10.1145 / 1041791.1041793$

[15] OSTIGUY, J.-F., NG, K.-Y. Optimized wakefield computations using a network model. In Proceedings of the XX International Linac Conference (LINAC). Monterey (California, USA), 2000, p. 275-277. OAI: CiteSeerX.psu:10.1.1.262.575

[16] DONGARRA, J. J., DUFF, I. S., SORENSEN, D. C., VAN DER VORST, H. A. Numerical Linear Algebra for High-Performance Computers. Philadelphia (Pennsylvania, USA): SIAM, 1998. ISBN: 0898714281. DOI: $10.1137 / 1.9780898719611$

[17] MEMON, N. M., AHMED, M. M., REHMAN, F. A comprehensive four parameters $I-V$ model for GaAs MESFET output characteristics. Solid-State Electronics, 2007, vol. 51, no. 3, p. 511-516. ISSN: 0038-1101. DOI: 10.1016/j.sse.2006.12.011
[18] MEMON, Q. D., AHMED, M. M., MEMON, N. M., RAFIQUE, U. An efficient mechanism to simulate DC characteristics of GaAs MESFETs using swarm optimization. In Proceedings of the IEEE 9th International Conference on Emerging Technologies (ICET). Islamabad (Pakistan), 2013, p. 1-5. DOI: 10.1109/ICET.2013.6743542

[19] DOBEŠ, J., POSPÍŠIL, L. Enhancing the accuracy of microwave element models by artificial neural networks. Radioengineering, 2004, vol. 13, no. 3, p. 7-12. ISSN: 1210-2512

[20] DOBEŠ, J., GRÁBNER, M. Novel HEMT models with improved higher-order derivatives and extracting their parameters using multibias $s$-parameters. In Proceedings of the 33rd IEEE Compound Semiconductor Integrated Circuit Symposium (CSICS). Waikoloa Village, Big Island (Hawaii, USA), 2011, p. 189-192. DOI: 10.1109/CSICS.2011.6062468

[21] DOBEŠ, J., MÍCHAL, J., BIOLKOVÁ, V. Multiobjective optimization for electronic circuit design in time and frequency domains. $\mathrm{Ra}$ dioengineering, 2013, vol. 22, no. 1, p. 136-152. ISSN: 1210-2512

[22] DOBEŠ, J., MÍCHAL, J., PAŇKO, V., POSPÍŠIL, L. Reliable procedure for electrical characterization of MOS-based devices. Solid-State Electronics, 2010, vol. 54, no. 10, p. 1173-1184. ISSN: 0038-1101. DOI: $10.1016 /$ j.sse.2010.05.034

[23] DOBEŠ, J., ČERNÝ, D., YADAV, A. A more efficient arrangement of the sparse LU factorization for the large-scale circuit analysis. In Proceedings of the IEEE/IFIP 19th International Conference on VLSI and System-on-Chip (VLSI-SoC). Hong Kong (China), 2011, p. 416-421. DOI: 10.1109/VLSISoC.2011.6081619

[24] AVAGO TECHNOLOGIES ATF-54143 Low Noise Enhancement Mode Pseudomorphic HEMT in a Surface Mount Plastic Package (datasheet). 16 pages. [Online] Cited 2012-06-08. Available at: http://www.avagotech.com/docs/AV02-0488EN

[25] AGILENT TECHNOLOGIES, INC. ADS 2008 Nonlinear Devices (datasheet). 728 pages. [Online] Cited 2008-01-31. Available at: http://cp.literature.agilent.com/litweb/pdf/ads2008/pdf/ccnld.pdf

[26] ALIAKBARI, H., ABDIPOUR, A., MIRZAVAND, R. Accurate timedomain modeling of multi-finger pHEMT transistor based on transmission line theory. AEÜ-International Journal of Electronics and Communications, 2015, vol. 69, no. 1, p. 215-225. ISSN: 1434-8411. DOI: $10.1016 /$ j.aeue.2014.09.007

[27] CAPPY, A. Noise modeling and measurement techniques. IEEE Transactions on Microwave Theory and Techniques, 1988, vol. 36, no. 1, p. 1-10. ISSN: 0018-9480. DOI: 10.1109/22.3475

[28] AVAGO TECHNOLOGIES A 802.11a WLAN Driver Amplifier using Enhancement Mode PHEMT ATF-54143 Transistor (datasheet). 8 pages. [Online] Cited 2010-07-14. Available at: http://www.avagotech.com/docs/5988-5845EN

\section{About the Authors ...}

Josef DOBEŠ received the Ph.D. degree in Microelectronics from the Czech Technical University in Prague in 1986. From 1986 to 1992, he was a researcher of TESLA Research Institute. He is currently with the Department of Radioelectronics of the Czech Technical University in Prague. His current research interests include physical modeling of elements of electronic circuits, especially radio-frequency and microwave transistors and transmission lines, creating or improving special algorithms for circuit analysis and optimization such as time- and frequency-domain sensitivity, poles-zeros or steady-state analyses, and creating a CAD tool for analysis and optimization of radio-frequency circuits. 
Jan MíCHAL received his M.Sc. degree in Electrical Engineering from the Czech Technical University in Prague in 1993. He received the Ph.D. degree in Radio Engineering at the Czech Technical University in Prague in 1986. Currently, he is affiliated with the Department of Radioelectronics of the Czech Technical University in Prague. His research interests mainly consist in the numerical methods for integrated as well as discrete RF circuit analysis and synthesis, especially using optimization methods, and practical methods for designing the RF circuits.

Jakub POPP was born in Prague (Czech Republic) in 1990 and lives in South Bohemia. He is a student of Czech Technical university, studies a M.Sc. in Wireless Communications since the last year and cooperates with the Department of Radioelectronics of the Czech Technical University. His current research interests are design $\mathrm{RF}$ circuits and utilization of terrestrial systems for navigation and location.

David ČERNÝ was born in Prague, Czech Republic, in 1985. He received his M.Sc. degree in 2009 from the Faculty of Electrical Engineering of the Czech Technical University in Prague. Since September 2009 he has been a PhD student at the Department of Radioelectronics at Czech Technical University in Prague. His research interests include simulation of high frequency circuits, physical modeling of electrical devices, and very large-scale integrated circuit analysis.

Martin GRÁBNER was born in Prague, the Czech Republic, in 1976. He received his M.Sc. and Ph.D. degrees in Electrical Engineering from the Czech Technical University in Prague, the Czech Republic, in 2000 and 2008, respectively. $\mathrm{He}$ is a researcher at the Dept. of Frequency Engineering of the Czech Metrology Institute in Prague. His research activities are in the field of electromagnetic wave propagation for terrestrial communications in the frequency band from $1 \mathrm{GHz}$ to $\mathrm{nm}$ wavelengths of free space optical lines.

František VEJRAŽKA gained his Ph.D. degree from Czech Technical University in Prague, Czech Republic, in 1972. He is the full professor of radio navigation, radio communications, and signals and systems theory. His main professional interest is in the radio satellite navigation. His team at the Faculty has been the provider of the experimental DGPS reference station which has disseminated DGPS corrections through the FM RDS and VLF systems. He has been principal manager of the project of the Ministry of Transport participation in the project "GALILEO." At present, he is principal manager of the project "Center of Competence: Integrated Satellite and Terrestrial Navigation Technologies Center."

Jakub KÁKONA was born in Cheb (Czech Republic) in 1988. He is a post gradual student of Czech Technical University in Prague at Department of Radioelectronics. He holds M.Sc. in Aerospace Systems. His current research interests are radioastronomy, navigation and passive radar systems.

Štěpán MATĚJKA was born in 1973. He received his Ph.D. from the Faculty of Electrical Engineering, Czech Technical University in Prague in 2004. From 1996 to 1997 he was with Elektrotechnika Tesla Kolín, Czech Republic, where he worked as a development engineer and cooperated on finalization of GPS based on board navigation system. In 2000, he affiliated the Czech Technical University in Prague as a radio frequency measurement and design tutor. His research interests include radio frequency circuits and systems design and measurement, digital signal processing, and radio transmitters and receivers systems. 